\title{
A demonstration of mobile phone deployment to support the treatment of acutely ill children under five in Bushenyi district, Uganda.
}

\author{
Jerome Kabakyenga ${ }^{1}$, Celestine Barigye ${ }^{2}$, Jennifer Brenner ${ }^{3}$, Samuel Maling ${ }^{1}$, Denise Buchner ${ }^{3}$, \\ Alberto Nettle-Aquirre ${ }^{3}$, Nalini Singhal ${ }^{3}$, Teddy Kyomuhangi ${ }^{1}$, David Tumusiime ${ }^{1}$, \\ Janet Finch ${ }^{4}$, Stuart MacLeod ${ }^{4}$
}

1. Mbarara University of Science and Technology, Uganda

2. Ministry of Health, Uganda

3. Faculty of Medicine, University of Calgary

4. Child \& Family Research Institute, University of British Columbia

\begin{abstract}
:
Background: Benefits of mobile phone deployment for children $<5$ in low-resource settings remain unproven. The target population of the current demonstration study in Bushenyi District, Uganda, presented with acute fever, pneumonia, or diarrhoea and were treated by community health workers (CHWs) providing integrated community case management (iCCM). Methods: An observational study was conducted in five parishes (47 villages) served by CHWs well versed in iCCM with supplemental training in mobile phone use. Impact was assessed by quantitative measures and qualitative evaluation through household surveys, focus group discussions, and key informant interviews.

Results: CHWs in targeted sites improved child healthcare through mobile phone use coupled with iCCM. Of acutely ill children, 92.6\% were correctly managed. Significant improvements in clinical outcomes compared to those obtained by CHWs with enhanced iCCM training alone were unproven in this limited demonstration. Nonetheless, qualitative evaluation showed gains in treatment planning, supply management, and logistical efficiency. Provider confidence and communications were enhanced as was ease and accuracy of record keeping.

Conclusion: Mobile phones appear synergistic with iCCM to bolster basic supportive care for acutely ill children provided by CHWs. The full impact of expanded mobile phone deployment warrants further evaluation prior to scaling up in low-resource settings.

Keywords: mobile phone deployment, ill children under five, Bushenyi district, Uganda.

DOI: http://dx.doi.org/10.4314/ahs.v16i1.12

Cite as: Kabakyenga J, Barigye C, Brenner J, Maling S, Buchner D, Nettle-Aquirre A, Singhal N, Kyomuhangi T, Tumusiime D, Finch J, MacLeod S. A demonstration of mobile phone deployment to support the treatment of acutely ill children under five in Bushenyi district, Uganda. Afri Health Sci. 2016;16(1): 89-96. http://dx.doi.org/10.4314/ahs.v16i1.12
\end{abstract}

\section{Background}

Well-trained and equipped volunteer community health workers (CHWs) can play a significant role in minimizing child mortality and morbidity in Uganda. In the face of severe constraints on healthcare resources, attention must focus on cost-effective interventions with potential to improve quality of acute care and timeliness of referral for acutely ill children under five years of age. Globally, a program of integrated community case management (iCCM), involving assessment and delivery of simple treatments by lay CHWs for fever, presumed pneumonia and diarrhoea has supported more

\section{Corresponding author: \\ Stuart MacLeod, \\ Child \& Family Research Institute, \\ University of British Columbia \\ Email:smacleod@cfri.ca}

effective illness management in rural settings. ${ }^{1,2}$ The approach is derived from the 2005 World Health Organization (WHO) strategy for integrated management of childhood illness (IMCI). ${ }^{3}$ Uganda is actively engaged in the introduction of $\mathrm{iCCM}^{1,2}$ and now proposes national scale up as a key strategy to address high rates of child mortality. ${ }^{4}$

Infection in young children is one of the greatest worldwide public health challenges, accounting for many of the 6.3 million, frequently preventable deaths in under five years of age reported in $2013 .^{5}$ As a parallel dilemma in Africa, there are significant shortages of well-trained health professionals and allied caregivers to deliver critical treatment when children fall ill. ${ }^{6-8}$ Task shifting, using lay trained CHWs to treat leading causes of mortality, has been proposed as a strategy to potentially reduce under five deaths in sub-Saharan Africa. ${ }^{6-8}$ Success with this intervention will introduce expanded 
roles for CHWs in assessment and treatment of acutely ill children. Such an approach should improve access by patients and families to antimalarials for fever, antibiotics for pneumonia, and oral rehydration salts (ORS) and zinc for diarrhea.'

In Uganda, CHWs often have limited education ${ }^{10}$ and iCCM delivery is further challenged by the remoteness of communities, logistics of program operations, ${ }^{11}$ erratic data management, unreliable drug availability, and limited monitoring to ensure that treatment algorithms are accurately followed. ${ }^{12}$ Furthermore, the information base designed to guide standardized treatment through CHW interventions is often poorly accessible. Groups developing iCCM programs have proposed that mobile phones should prove valuable ${ }^{13}$ and the potential for deployment to strengthen healthcare delivery is widely recognized as a priority research question. ${ }^{14-16}$

Another concern in isolated settings has been the availability of essential medicines for children, addressed in part by WHO initiatives since 2007. ${ }^{17-20}$ WHO has embraced a comprehensive program aimed at improving essential medicines access and augmenting the information base and dissemination of standardized therapeutic guidelines for acutely ill young children. The expanding use of improved communication systems and mobile phone technology holds promise of addressing issues such as erratic anticipation of drug supply needs in rural settings. ${ }^{21}$

Globally, health programs have incorporated mobile(M)-health to support improved care delivery ${ }^{22}$ and this holds promise for Uganda where rapid development of wireless communication has occurred. ${ }^{23} \mathrm{Mo-}$ bile phones have been widely used in recent years to enhance data management, ${ }^{24}$ and improve communication among providers. ${ }^{25}$ Positive influences on health have been shown at a system level in low-resource settings. ${ }^{26}$ Phones have been demonstrated to have significant impact on adherence to therapeutic regimens and on anti-infective clinical outcome indicators in countries such as Kenya. ${ }^{27}$ Although M-health programs have proliferated throughout sub-Saharan Africa there remains little community-level documentation of their overall utility in improving specific health outcomes. ${ }^{28-31}$.

This paper describes a community-based demonstration project undertaken to explore whether mobile phones used by volunteer CHWs as an adjunct to iCCM might improve the management of acutely ill children under five in Bushenyi District, SouthWestern Uganda. The project sought complementary gains in health promotion and disease prevention for mothers and children as previously reported by Healthy Child Uganda. ${ }^{32-34}$

\section{Methods}

As presented in Figure 1, nine parishes in Kyabugimbi sub-county were selected as sites for advanced CHW training in $\mathrm{iCCM}$ as per the protocol of a controlled intervention trial ${ }^{35}$ building on previous studies of $\mathrm{CHW}$ engagement in iCCM..$^{32-34}$ The choice of sites was based on the burden of childhood illness in this sub-county and on difficulty of access to the nearest level IV health centre. In the Ugandan healthcare system, level IV centres are designed to provide medical coverage for an average population of 100,000 people within a county. The centres include ward beds, an operating theatre, laboratory and blood transfusion facilities. ${ }^{36}$

In 98 villages participating in the background trial, ${ }^{35}$ selected CHWs $(n=196)$ received 5-day basic training followed by additional iCCM preparation. Training in iCCM focused on skills and knowledge needed to assess, treat and, if necessary, refer to a higher level of care, young children with fever, presumed pneumonia or diarrhoea.

Five parishes were randomly selected (names drawn from a hat) as demonstration sites for the introduction of a mobile phone support system as reported here. In 47 communities within the selected parishes, $96 \mathrm{CHWs}$ who had completed iCCM basic and advanced preparation received supplementary training, equipment and follow-up to enable their use of mobile phones augmenting iCCM. ${ }^{30}$ The introduction of mobile phone support occurred in two phases: (1) a nine-month preliminary refinement of procedures and CHW training, and (2), a seven-month study period with real time data collection.

In view of the small study size and short observation period, both qualitative and quantitative methods were used to evaluate the potential benefits of mobile phone support. Quantitative measures included accuracy of diagnosis and treatment, early recognition of danger signs, and appropriateness and timeliness of referral to suitable levels of care. Qualitative measures included: (i) perceptions of improved connectivity to other CHWs and to healthcare workers at higher levels of care; (ii) self-reported $\mathrm{CHW}$ assurance in quality of assessments of acutely ill children; (iii) perceived ease of data management; (iv) confidence in ability to avoid essential medicine stockouts. 
Ethical approval was obtained from all relevant review boards in Uganda and Canada with a consent process consistent with best practices in settings with low literacy.

Figure 1: Distribution of population included in demonstration project: Bushenyi District

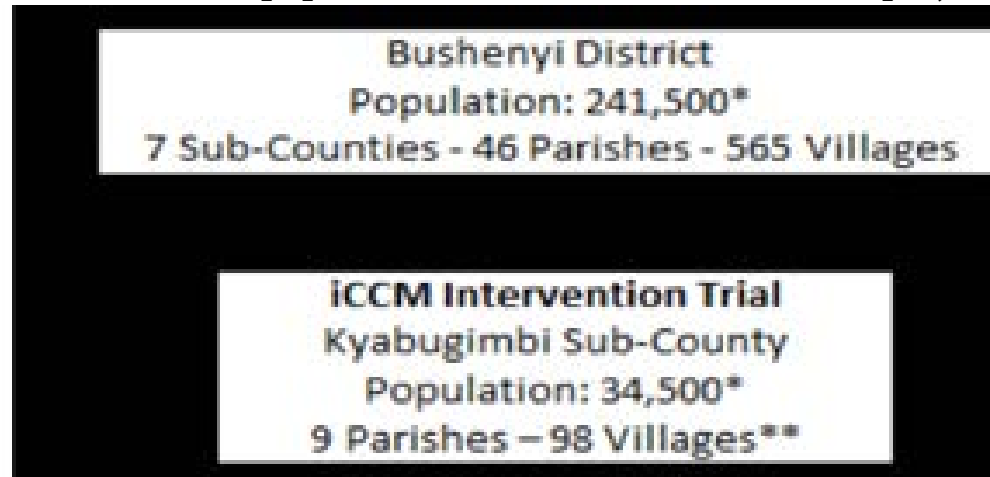

KCCMA plus anobile demonstration project

5 parishes, 47 villages

randomly selected $Q$ WWs received additions

iCOM + mobile phone training *...

$\mathbf{n}=\mathbf{9 6}$

\section{Post intervention random selection of households \\ to be surveyed in each village: total $n=\pi 0$}

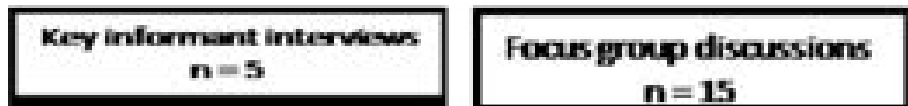

*estimates for 2010 obtainol from the Bushervi databases propectod from 2002 Uganxh national

census

Dats

** reference [35]

** reference $[30 \mid$

Quantitative methods

\section{(i) Patient encounter reports}

During basic training all CHWs were instructed in record keeping. Each was issued a government-approved register to record basic demographic and health indicators related to households and individuals. 'iCCM CHWs' were additionally trained to record each sick child encounter, noting name, age, sex, village, illness signs, breathing rate, danger signs, treatment and referrals.

Beyond recording encounters in registers, 'iCCM plus mobile' CHWs entered information on their mobiles following iCCM algorithm prompts. Data from this cohort was automatically downloaded to a server at Kyabugimbi health centre ${ }^{30}$ and was available for comparison with paper reports. Analysis focused on frequencies and treatment choices between April 1 and October 31, 2012 and recorded treatment as 'appropriate' if it was in accordance with the iCCM algorithm. The following, with dosage adjustments, were deemed 'appropriate':artemether-lumefantrine (Coartem ${ }^{\circledR}$ ) for children 4-59 months old with fever, amoxicillin for children 2-29 months old with cough and rapid breathing, ORS and zinc for children 2-59 months old with diarrhea. ${ }^{35}$

\section{(ii) Household surveys}

As described by Buchner and colleagues ${ }^{37}$ a household survey of mothers of young children was conducted in November 2012 in 15 randomly selected households per village. The tool was translated into the local language, back-translated, and field-tested prior to data collection. ${ }^{37}$ Trained research assistants not involved in the study intervention administered surveys verbally. Treatment was determined 'appropriate' if the mother reported that the child received, in age-appropriate dosage, artemether-lumefantrine (Coartem $\left.{ }^{\circledR}\right)$ ) for fever, an antibiotic for cough and rapid breathing, and ORS and zinc for diarrhoea. 


\section{Qualitative methods}

Focus group discussions (FGDs) and key informant interviews (KIIs) were conducted after October 31, 2012 under supervision (co-author DB). FGD and KII questionnaire tools were semi-structured, developed in English, translated into local dialect (Runyankore) and then back-translated. Questionnaires surveyed impressions of iCCM: access to care, perceived confidence in $\mathrm{CHW}$ services, satisfaction with quality of care, referral, patient encounters, supervision and drug supply. ${ }^{37}$

FGDs and KIIs were conducted following at least seven months of iCCM intervention coupled with mobile phone deployment. Participants were caregivers, CHWs, health centre staff, local leaders and government health officials. To ensure a representative sample, FGD par- ticipants were selected using Rand's random digit sampling. ${ }^{38}$

Experienced facilitators native to western Uganda conducted FGDs in local language. KIIs were conducted in either English or Runyankore based on participant preference. All FGDs and KIIs were audio recorded; transcribed and translated as required into English. Qualitative results were analyzed by co-author DB. ${ }^{37}$

\section{Results}

The demonstration project which is the subject of this paper began only after CHWs had mastered mobile phone usage with supporting software for health purposes. ${ }^{30}$ The education qualifications of the 60 female and 36 male CHWs provided with mobile phones and trained in their clinical use are shown in Table 1.

Table 1: Educational qualification of $\mathrm{CHWs}$ receiving mobile phone training as a supplement to extended iCCM preparation.

\begin{tabular}{|l|l|}
\hline Total number of CHWs trained & 96 \\
\hline Educational level achieved & \\
Primary 6 or less & 9 \\
Primary 7 & 42 \\
Senior 1 & 4 \\
Senior 2 & 12 \\
Senior 3 & 7 \\
Senior 4 & 19 \\
Tertiary/college up to senior 6 & 3 \\
\hline
\end{tabular}

Descriptive quantitative data provided some evidence of potential value for mobile phone support. During the seven study months for which operational data was analyzed, a total of 4,552 cases of acute illness were dealt with by CHWs with mobile phones. Of these cases, 2,033 were classified as fever, 2,087 as 'presumed pneumonia' and 432 as diarrhoea. The CHWs supported by mobile phones instituted 'appropriate' treatment in $97.1 \%$ of fever cases, $88.2 \%$ of pneumonia cases and $92.4 \%$ of diarrhoea cases, based on the treatment algorithms which are a component of iCCM training. As shown in table 2, CHWs achieved a high rate of appropriate treatment overall $(92.6 \%)$.

Table 2: CHW assessment and treatment by case, showing illness classification and percentage 'appropriate' treatment given according to $\mathrm{CHW}$ patient encounter reports

\begin{tabular}{|l|l|l|}
\hline Classification & $\begin{array}{l}\text { \# cases } \\
\text { iCCM/mobile sites* }\end{array}$ & $\begin{array}{l}\% \\
\text { treatment** }\end{array}$ \\
\hline fever $^{1}$ & 2033 & 97.1 \\
\hline presumed pneumonia $^{2,3}$ & 2087 & 88.2 \\
\hline diarrhea $^{2}$ & 432 & 92.4 \\
\hline all Cases & 4552 & 92.6 \\
\hline
\end{tabular}

A 'case' occurs each time a CHW classifies an illness in a child, according to the ICCM algorithms; a single child assessed may be assigned to more than one category of illness; therefore number of cases of illness may exceed total number of assessments by CHWs.

* case numbers treated by CHWs with iCCM training and mobile phone support

** 'appropriate' treatment is defined as CHW reported medication/treatment in agreement with iCCM algorithm for classified illness and age range.

${ }^{1}$ cases in 4-59 month-old children

2 cases in 2-59 month-old children

${ }^{3}$ presumed pneumonia $=$ cough + rapid breathing 
The study of mobile phone deployment was a parallel component of a controlled intervention trial of iCCM as implemented by CHWs. Consequently, descriptive comparison data is available ${ }^{35}$ although not collected in a form suitable for statistical analysis. Trained CHWs without mobile phone support achieved appropriate treatment of fever in $93.6 \%$ of 1692 cases, of presumed pneumonia in $92.3 \%$ of 1805 cases and of diarrhoea in $90.4 \%$ of 291 cases. While the clinical management appears comparable, no definitive evidence of improvedoutcome could be obtained in this study design.

Observational quantitative data provided evidence of successful implementation of the mobile phone support system. All 96 trained CHWs proved capable of using the technology. Submission of 4,552 case reports via mobile phone provided an opportunity for an immediate check on the accuracy and completeness of records and correction of any errors. This represented a dramatic improvement over written registers which were often completed after considerable delay and only available for review at monthly meetings. Mobile phone records indicated that $68.5 \%$ of acutely ill children were seen within 24 hours. Over the seven month study period, there were 72 requisitions for resupply of medicines submitted through a short messaging system and identified needs were rapidly met. There were five records of incorrect drug administration identified. No stockouts were reported during the study period.

The project's end line survey of maternal self-reports also provided quantitative evidence of a desirable pattern of care delivered for children reporting symptoms of fever or cough with rapid breathing in the communities provided with iCCM and mobile phone support. In the mobile phone parishes overall, $68 \%$ of affected children received treatment within the public system. Among those who actively sought care, $71 \%$ were treated by CHWs and a further $7 \%$ received treatment at the public health centre. The remaining $22 \%$ accessed care through private centres, pharmacies, shops and traditional healers. This care pattern, showing a high level of reliance on the public system, is a positive finding.

The most significant observations about mobile phone support emerged in qualitative inputs from FDGs and KIIs. Both CHWs and higher level healthcare workers applauded the potential provided by mobile phones for improved quality assessment and treatment of sick children. The CHWs were able to follow the algorithms on their phones and this increased their confidence in the care they could provide. A CHW stated 'In case a child gets a dangerous sign...it is easy to call the in-charge and she helps me on how to treat'.

The level IV health centre staff was able to check decisions immediately and to recommend revised or corrected treatment as necessary. One healthcare worker reported that 'The mobile phone helps us to treat well and allows us to correct them (CHWs) in case of a mistake instead of seeing the mistakes at the end of the month'. The mobile phone system facilitated referrals through two-way communication and calls made prior to transfer. One CHW reported 'We could call and tell the HC staff about the patient we were about to send....the HC staff would be there waiting'. Another CHW said 'Young children below five years - we no longer take them to Kyabugimbi because most diseases like cough and high fever are now being treated here by me'.

Connections within CHW teams were strengthened and a sense of community support and friendship was created though interactions to obtain peer advice about patients as well as technical advice. One CHW reported 'It is easier to alert our fellow CHWs on any matter concerning the health of the local community. It has also helped that we are able to communicate with the chairman in the case of a meeting'.

The improvement in medicine supply chain management was perceived as important by CHWs. Checks on supplies were consistent and replenishment was efficient. Transport costs were reduced. A CHW reported 'The mobile phones have helped us connect with our bosses in requesting more medicine....we don't use our money for transport in order to collect the medicine... just call and the medicines are brought to your doorstep'.

Perhaps the most important qualitative observation relates to ease of data collection and advantages of timely data management. The information about acute illness is reported in real time and because it is submitted electronically, collecting and sorting of data by HC staff is facilitated. The advantage over paper registers was notable. One CHW reported 'They (iCCM registers) are time consuming while the mobile phone is quick and easy'. A district health leader stated 'The beauty in this is that the message is instant to the consumer...this will 
now mean that we can receive information in real time and that will trigger the next step of action'.

\section{Discussion}

This demonstration, while limited in scope, has indicated the advantages achievable through a partnership that includes CHWs, healthcare workers within the public system, academic child health specialists, communication technology experts, including private sector service providers, and health system administrators. Challenges met and overcome in development and implementation of such a system have been previously published. ${ }^{30}$

The short duration of the demonstration and relatively infrequent occurrence of severe illness precludes definitive conclusions about overall health outcomes or clinical benefits that may eventually prove attributable to mobile phone support. End of project survey results demonstrated a significant increase in the proportion of acutely ill children being assessed and appropriately treated both in communities provided with enhanced iCCM alone and in communities provided with iCCM plus mobile phone support. Overall, the results reinforce the value of a robust iCCM system in facilitating parental decisions to seek medical treatment when available close to home.

A compelling lesson has been the impact of a mobile phone system on the collection of real time data. The ability of CHWs to manage an average of 6.7 cases per month was enhanced through mobile phone system supports. The improved accuracy in measuring case prevalence and related demand for drug treatments has been universally welcomed by healthcare workers and administrative leaders. Improved communication enhanced supply chain management of essential medicines and enabled sharing of medicines between CHWs and across parish boundaries. As a result of rapid communication, the supporting level IV health facility was better able to manage supplies of essential medicines. No stockouts occurred during the course of the study although these had previously been a common occurrence.

A National iCCM program as proposed and planned by the Ugandan Ministry of Health currently involves provision and maintenance of cumbersome $\mathrm{CHW}$ registers documenting patient encounters. These record books are relatively costly, cumbersome to update, require frequent replacement and are often subject to inaccuracy. Health centre and district health programmers report- ed great advantages in collecting and sorting data input from the mobile phone system. The operational benefit conferred by mobile phone support should prove cost-effective if a paperless system can be maintained.

Observations to date of system function at a district, sub-county and parish level indicate value added through improved integration to serve patient management priorities and to facilitate sharing of patient information and treatment guidelines. The hub created in this project promises to contribute to improved maintenance of drug supplies and to future learning by health caregivers at all levels.

In this limited observational study, no demonstrable improvement in health outcomes attributable to mobile phone support has been shown. However, qualitative evidence indicates that worthwhile improvements in operational efficiency are achievable through wider mobile phone deployment technology.

\section{Conclusions}

The demonstration project described featured introduction of mobile-phone-enabled system with potential to improve iCCM efficiency for acutely ill children under five years of age in a rural district. Mobile phones work synergistically with iCCM to bolster the supporting care of CHWs. The qualitative data reported also indicate that mobile phone support is an important element in building trust among volunteers and in strengthening their working relationship with healthcare workers at local health centres. In addition, within the context of this study, the timelines for case reporting were greatly improved with mobile phone support, which points to their utility and potential to reduce costs of cumbersome paper-based registries.

The demonstration project has shown a high level of acceptance of mobile phone technology as an adjunct to the provision of child health services at all levels within the Bushenyi District and in its adjacent academic health science centre at Mbarara University of Science and Technology. Beginning with the emphasis on training of CHWs in iCCM in accordance with the Ministry of Health policy for Bushenyi District, it has been shown through qualitative reporting that the supports provided through a mobile phone system may create operational efficiencies. The gains achieved by iCCM amplified by mobile phone support are complementary to those previously described by the Healthy Child Uganda partnership. ${ }^{32-34}$ 
The Uganda Ministry of Health is considering the scaling up of the mobile phone enabled system described in this paper in order to strengthen management of acute conditions causing preventable morbidity and mortality in children below five years of age.

\section{Acknowledgments}

JK, DT, JF and SM wrote the first draft of this paper. All authors contributed to the planning and execution of the demonstration project and linkage of the mobile phone study to the parent study of iCCM implementation. All authors contributed to editing and completion of the manuscript. We are grateful to the many community health workers, trainers, health centre staff, community members and leaders whose input has been critically important. Participation of families in the study parishes is gratefully acknowledged. The research team has been supported throughout staff and officers from Mbarara and Bushenyi health services and the national Ministry of Health (Jesca Nsungwa Sabiiti). Dr Matthew Wiens (University of British Columbia) has provided valuable logistical support in Mbarara. Financial support provided by Canada's International Development Research Centre (grant 106379-002).

\section{References}

1. Marsh DR, Hamer DH, Pagnoni F, Peterson S. Introduction to a special supplement: Evidence for the implementation, effects, and impact of the integrated community case management strategy to treat childhood infection. American Journal of Tropical Medicine and Hygiene 2012; 87(Suppl 5): 2-5.

2. WHO/UNICEF Joint Statement: Integrated Community Case Management (iCCM). An equity-focused strategy to improve access to essential treatment services for children. Available: http://www.unicef.org/ health/files/iCCM_Joint_Statement_2012.pdf cessed 22 May 2015)

3. World Health Organization: Maternal, newborn, child and adolescent health. Documents on the integrated management of childhood illness (IMCI).http://www. who.int/maternal_child_adolescent/documents/imci/ en/index.html (accessed 22 May 2015)

4. Project brief. ICCM in peri-urban villages in Uganda. http://comdis-hsd.dfid.gov.uk/research-themes/ community-interventions/iccm-in-peri-urban-villagesin-uganda (accessed 22 May 2015)

5. World Health Organization Children: reducing mortality. Fact sheet 178. September 2014. http://www. who.int/mediacentre/factsheets/fs178/en/ (accessed 22 May 2015)
6. Anywangwe SCE, Mtonga C. Inequities in the global health workforce: The greatest impediment to health in sub-Saharan Africa. International Journal of Environmental Research andPublic Health 2007; 4: 93-100.

7. Chopra M, Munro S, Lavis JN, The McKinsey Quarterly 2008; 1: 98-105.

8. Conway MD, Gupta S, Khajavi K. Addressing Africa's health workforce crisis. The McKinsey Quarterly 2008; 1: 98-105.

9. UNICEF, Uganda Ministry of Health, WHO. Kampala, Uganda May 2010: Integrated community case management of childhood malaria, pneumonia and diarrhoea. Implementation guidelines. https://www. $\mathrm{k} 4$ health.org/sites/default/files/ICCM\%20Implementation\%20Guidelines.pdf (accessed 22 May 2015)

10. Haines A, Sanders D, Lehmann U, et al. Achieving child survival goals: potential contribution of community health workers. Lancet 2007; 369: 2121-2131.

11. Bhutta AZ, Lassi ZS, Pariyo G, Huicho L. World Health Organization. Global Health Workforce Alliance. Global experience of community health workers for delivery of health related millennium development goals: a systematic review, country case studies, and recommendations for integration into national health systems.http://www.who.int/workforcealliance/knowledge/publications/CHW_FullReport_2010.pdf?ua=1 (accessed 22 May 2015)

12. Oluwole D, Mason E, Costello A. Management of childhood in Africa. Early evaluations show promising results. British Medical Journal 2000; 320: 594-595.

13. Strachan DL, Kallander K, Ten Asbroek AH, et al. Improved motivation and retention of community health workers delivering integrated community case management (iCCM): Stakeholder perceptions and priorities. American Journal of Tropical Medicine and Hygiene 2012; 87(Suppl 5): 111-119.

14. Hamer DH, Marsh DR, Peterson S, Pagnoni F. Integrated Community Case Management: Next steps in addressing the implementation research agenda. American Journal of Tropical Medicine and Hygiene 2012; 87(Suppl 5): 151-153.

15. Berkley S. How cell phones are transforming health care in Africa. MIT Technology Review 2013. http:// www.technologyreview.com/view/519041/how-cellphones-are-transforming-health-care-in-africa/ (accessed 22 May 2015)

16. Wesolowski A, Eagle N, Tatem AJ, et al. Quantifying the impact of human mobility on malaria. Science 2012; 338: 267-270.

17. World Health Organization. 60th World Health Assembly Resolution 23 May 2007: World Health Assem- 
bly 60.20: Better medicines for children.http://www. who.int/gb/ebwha/pdf_files/WHA60/A60_R20-en. pdf (accessed 22 May 2015)

18. World Health Organization: Model list of essential medicines for children. http://www.who.int/medicines/publications/essentialmedicines/4th_EMLc_FINAL_web_8Jul13.pdf (accessed 22 May 2015)

19. World Health Organization Model Formulary for Children 2010. http://www.who.int/selection_medicines/list/WMFc_2010.pdf (accessed 21 Nov 2014)

20. Macleod SM, Finch JK, Macharia WM, Anabwani GM. Better drug therapy for the children of Africa: current impediments to success and potential strategies for improvement. Pediatric Drugs 2013; 15: 259-269.

21. Tashobya CK, Ssengooba F, Oliveira-Cruz V, editors. Health Systems Reforms in Uganda: processes and outputs. eResearch: the open access repository of the research output of Queen Margaret University, Edinburgh. http://eresearch.qmu.ac.uk/762/1/eResearch_762.pdf (accessed 22 May 2015)

22. Geneva Dialogue on $\mathrm{m}$-health. Using mobile technology to advance public health in developing world. International Telecommunications Union News July/ August2011.http://www.itu.int/net/itunews/issues/2011/06/42.aspx (accessed 22 May 2015)

23. Siedner M, Haberer J, Bwana M, Ware NC, Bangsberg DR. High acceptability for cell phone text messages to improve communication of laboratory results with HIV-infected patients in rural Uganda: a cross-sectional survey study. BMC Medical Informatics and Decision Making 2012; 12: 56.

24. Tomlinson M, Solomon W, Singh Y, et al. The use of mobile phones as a data collection tool: A report from a household survey in South Africa. BMC Medical Informatics and Decision Making 2009; 9: 51.

25. Asiimwe C, Gelvin D, Lee E, et al. Use of an innovative, affordable, and open-source short message service-based tool to monitor malaria in remote areas of Uganda. American Journal of Tropical Medicine and Hygiene 2011; 85: 26-33.

26. Piette JD, Lun KC, Moura Jr LA, et al. Impacts of e-health on the outcomes of care in low- and middle-income countries: where do we go from here? Bulletin of the World Health Organization 2012; 90: 365-372.

27. Lester RT, Ritvo P, Mills EJ, et al. Effects of a mobile phone short message service on antiretroviral treatment adherence in Kenya WelTel Kenya1: a randomised trial. Lancet 2010; 376: 1838-1845.
28. Kaplan WA. Can the ubiquitous power of mobile phones be used to improve health outcomes in developing countries? Global Health 2006; 2: 9. doi:10.1186/1744-8603-2-9.

29. Rashid AT, Elder L. Mobile phones and development: an analysis of IDRC-supported projects. The Electronic Journal on Information Systems in Developing Countries 2009; 36: 1-16.

30. Tumusiime D, Agaba G, Kyomuhangi T, Finch J, Kabakyenga J, MacLeod S. Introduction of mobile phones for use by volunteer community health workers in support of $\mathrm{iCCM}$ in Bushenyi District, Uganda: Implementation process. BMC Health Services Research 2014; 14(Suppl 1): S2.

31. Lester RT, Gelmona L, Plummer FA. Cell phones: tightening the communication gap in resource-limited antiretroviral programmes. AIDS 2006; 20: 2242-2244. 32. Scholastic A, Khomuhangi K, Santarino D, MacDonald N, LeBlanc J. Healthy Child Uganda survey on knowledge, attitude and behaviour of village health team (VHT) members towards their responsibilities in southwestern Uganda. Pediatrics and Child Health 2012; 17: e89.

33. Ludwick T, Brenner JL, Kyomuhangi T, Wotton KA, Kabakyenga J. Poor retention does not have to be the rule: Retention of volunteer community health workers in Uganda. Health Policy and Planning 2014; 29(3): 388-395.

34. Brenner JL, Kabakyenga J, Kyomuhangi T, et al. Can volunteer community health workers decrease child morbidity and mortality in southwestern Uganda? An impact evaluation. PLOS ONE 2011; 6: e27997.

35. Brenner JL, Barigye C, Maling S, et al. Where there is no doctor: Can volunteer community health workers in rural Uganda provide integrated community case management? African Health Sciences (submitted for review 2015)

36. Rutebemberwa E, Pariyo G, Peterson S, et al. Utilization of public or private health care providers by febrile children after user fee removal in Uganda. Malaria Journal 2009; 8: 45.

37. Buchner DL, Brenner JL, Kabakyenga J, et al. Stakeholders' perceptions of integrated community case management by community health workers: A post-intervention qualitative study. PLOS ONE. 2014. DOI: 10.1371/journal.pone.0098610.

38. The R project for statistical computing. http:/ /www.r-project.org (accessed 22 May 2015) 